\title{
The 15-Year Evolution of the Thoracoscopic Anterior Release: Does It Still Have a Role?
}

\author{
Rattalerk Arunakul ${ }^{1}$, Alex Peterson ${ }^{2}$, Carrie E. Bartley², \\ Krishna R. Cidambi ${ }^{3}$, Eric S. Varley ${ }^{3}$, Peter O. Newton ${ }^{2,3}$ \\ ${ }^{I}$ Department of Orthopedic Surgery, Thammasat University, Bangkok, Thailand \\ ${ }^{2}$ Department of Orthopedic Surgery, Rady Children's Hospital and Health Center, San Diego, CA, USA \\ ${ }^{3}$ Department of Orthopedic Surgery, University of California San Diego, San Diego, CA, USA
}

Study Design: Retrospective.

Purpose: To determine how the indications for anterior thoracoscopic release and fusion have evolved over time.

Overview of Literature: Anterior release was commonly performed to correct severe spinal deformities before the advent of pedicle screw fixation. The thoracoscopic approach significantly reduced the morbidity, as compared to open thoracotomy procedures.

Methods: We reviewed charts and radiographs of pediatric spinal deformity patients who underwent thoracoscopic release/fusion for their deformity from 1994 to 2008. Indications for the thoracoscopic procedure were assigned to one of the following categories: hyperkyphosis, large/stiff scoliosis, crankshaft prevention, and 'other'. We analysed indications grouped in 3-year intervals to determine how the indications for this procedure evolved over the past 15 years.

Results: One hundred and thirty-eight patients (mean age, 15 years; range, 2-28 years) underwent the procedure, with 160 identified indications. The frequency of thoracoscopic anterior release/fusion decreased after peaking in the years 2000-2002. Initially, hyperkyphosis was the most frequent indication (15/33, 45\%; 1994-1996), but declined to an intermittent indication since 2006. The use of thoracoscopy to prevent crankshaft has also declined, but remains an indication for the most immature cases $(2 / 17,12 \%$; 2006-2008). Severe or rigid scoliosis is currently the most common indication for thoracoscopic release/fusion at our center (11/17, 65\%; 2006-2008).

Conclusions: The indications for a thoracoscopic anterior release/fusion has evolved with our increased understanding of this procedure and improved posterior fixation with pedicle screw instrumentation. Thoracoscopy in select spinal deformity patients still has an important role despite its less frequent use, as compared to the past decade.

Keywords: Scoliosis; Video-assited thoracoscopic surgery; Kyphosis; Spinal fusion; Endoscopic surgical procedures

\section{Introduction}

Mack et al. [1] and Rosenthal et al. [2] first reported patient outcomes for video-assisted thoracoscopy in various therapeutic procedures, including anterior release for scoliosis, discectomy, vertebral body biopsy, drainage of a disc space abscess, and anterior interbody fusion. Subsequently, this endoscopic technique was reported by numerous investigators for anterior thoracic and thoracolumbar reconstruction in cases of metastatic tumors,

Received Nov 28, 2014; Revised Jan 20, 2015; Accepted Jan 20, 2015

Corresponding author: Peter O. Newton

Department of Orthopedic Surgery, Rady Children's Hospital and Health Center,

3030 Children's Way, Suite 410, San Diego, CA 92123, USA

Tel: +1-858-966-6789, Fax: +1-858-966-7494, E-mail: pnewton@rchsd.org 
fractures, and deformities, as well as anterior release and fusion for the treatment of idiopathic scoliosis [3-9].

The successful development of the video-assisted thoracoscopic surgery technique for anterior scoliosis release, as well as the involved instrumentation significantly reduced the morbidity, as compared to open thoracotomy procedures [10]. Improvements in anterior approach techniques and instrumentation by Kostuik et al. [11] and Dwyer [12] in the late 1960s to mid 1970s proved superior to posterior surgerywith greater deformity correction and preserved motion segments [13]. However, with the advent of segmental pedicle screw fixation that enables more powerful corrective forces, it was suggested that an additional anterior procedure may be unnecessary even in severe deformities $[14,15]$.

We incidentally observed a decreased frequency of use during this transpedicular fixation era, hence we reviewed our experience and determined how the indications for anterior thoracoscopic release and fusion evolved over time.

\section{Materials and Methods}

We performed a retrospective single-center chart and radiographic review of pediatric spinal deformity patients who underwent a thoracoscopic anterior release/ fusion (with subsequent posterior instrumentation) from 1994-2008. Patients who received anterior instrumentation were excluded. Demograghic data, curve magnitude, radiographic skeletal maturity, operative data, and perioperative complication of patients were collected. A total of 138 patients who underwent an anterior thoracoscopic release and fusion procedure were identified between 1994-2008, with a total of 160 identified indications for the procedure. Demographic information was shown in Table 1. Of the 138 procedures, 119 (86\%) were right-sided thoracoscopic approach and 19 (14\%) were left-sided. There were a total of 877 excised intervertebral discs. The range of excised discs per patient was from 3 to 10 , with 6-8 discs excisions in the majority of patients (80\%). All patients had posterior fusion and instrumentation after completion of the anterior thoracoscopic procedure. The number of posterior fusion levels ranged from 4 to 17 , with a mode of 11 levels. The type of posterior instrumentation was classified as 1 of 2 groups: hook/pedicle screw construct versus sublaminar wiring. The hook/pedicle screw stystem was used in $117(85 \%)$ of the total cases
Table 1. Patient demographics

\begin{tabular}{lc} 
General data & Value \\
Age $(y r)(n=138)$ & $15 \pm 4(2$ to 28$)$ \\
Sex $(n=138)$ & $65(47)$ \\
Male & $73(53)$ \\
Female & $67 \pm 21(15$ to 110$)$ \\
Coronal Cobb $\left(^{\circ}\right)(n=97)$ & $48 \pm 17(14$ to 78$)$ \\
Side bending $\left(^{\circ}\right)(n=51)$ & $74 \pm 32(-39$ to 120$)$ \\
\hline Kyphosis Cobb $\left(^{\circ}\right)(n=55)$ & $47(55)$ \\
Triradiate cartilage $(n=86)$ & $39(45)$ \\
Closed & Mode $0(0$ to 5$)$ \\
Open &
\end{tabular}

Values are presented as mean \pm standard deviation (range) or number (\%).

from 1994 to 2008. The use of the sublaminar wiring system peaked at 12 cases in 1994-1996, declined thereafter, and was not used in any cases after 2000. The number of pedicle screws used increased substantially with the initiation of thoracic pedicle screws in 2001. Indications for the thoracoscopic anterior procedure were assigned to 1 of 4 categories: hyperkyphosis, severe or rigid scoliosis, crankshaft prevention, and 'other' (e.g., pseudoarthrosis prevention, thoracic lordosis). Subjects were not limited to 1 indication. Data were presented as grouped in 3-year intervals. Descriptive statistical analysis was performed to determine how the utility of this procedure has evolved over the past 15 years.

\section{Results}

From the first 3 years of the study period to the final 3 years, the average Cobb angle of patients undegoing this procedure increased from $60^{\circ}$ to $75^{\circ}$, while the average maximum kyphosis decreased from $77^{\circ}$ to $-19^{\circ}$. The distribution of diagnoses was presented in Fig. 1; idiopathic scoliosis (41\%), neuromuscular scoliosis (37\%), and Scheuermann kyphosis (21\%) were the most common. No hyperkyphotic patients underwent anterior thoracoscopic release and fusion in the final 3 years of the study period, as compared tothe higher frequency when the technique was first introduced.

While the number of spinal deformity surgeries performed at our institution per year has doubled since 1994, the frequency of thoracoscopic anterior release has decreased, since its peak between 2000-2002. Fig. 2 dis- 
played the frequency of the different indications for a thoracoscopic anterior spinal fusion at our center from 1994 to 2008. Hyperkyphosis was the most frequent indication (15/33, 45\%) between 1994-1996, but has since declined in frequency at our center with 0 patients undergoing a thoracoscopic release during the final 3 years of the study period. The use of thoracoscopy to prevent crankshaft also declined after reaching its peak at 14 (28\%) in 20002002 , but remains an indication for the most immature cases (2/17 indications, $12 \% ; 2006-2008)$. Severe (average $86^{\circ}, 70^{\circ}-110^{\circ}$ ) or rigid scoliosis is now the most common reason for performing a thoracoscopic release/fusion $(11 / 17,65 \%$; 2006-2008) at our center. In addition to hyperkyphosis, the 'other' indications (e.g., thoracic lordosis and pseudoarthrosis prevention) remain an intermittent indication at our center.

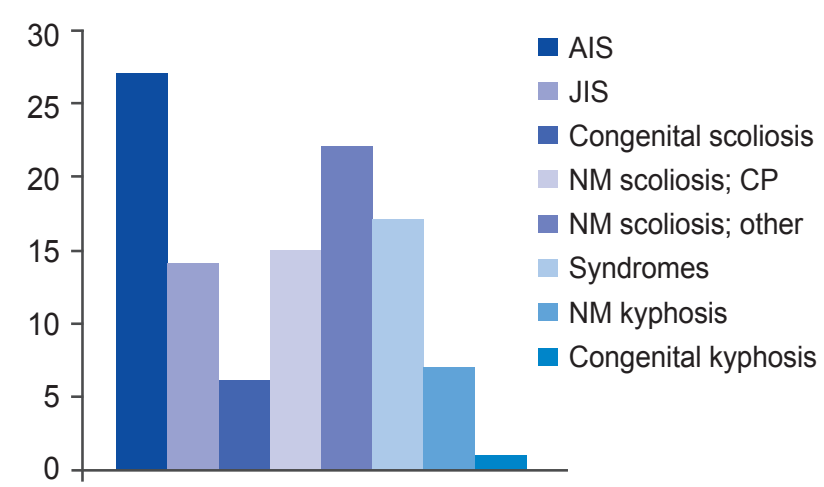

Fig. 1. Frequency of diagnoses of patients. AIS, adolescent idiopathic scoliosis; JIS, juvenile idiopathic scoliosis; NM, neuromuscular; CP, cerebral palsy.
A total of 44 postoperative complications were recorded between 1994 to 2008. Complications included 22 respiratory (16\%), 3 conversion to open-thoracotomy due to pleural adhesions that prevented lung retraction (2\%), 8 reoperations (6\%), 3 neurological (2\%), and 8 'other' problems (e.g., infection, cardiac arrhythmia, and device related; $6 \%$ ). The most common respiratory complications were atelectasis (12) and pleural effusions (8). Of the 22 respiratory complications, 4 required reintubation, 3 prolonged intubation, 3 chest tube placement, and 1 bronchoscopy. Indications for the reoperations included infection (4), pseudoarthrosis (1), misplaced hook (1), and curve progression (1). The neurological complications included 1 intraoperative neuromonitoring alert (transient loss of H-reflexes) and 2 incidences of paresthesia in the lower extremity (resolved by 2 weeks and 9 month postoperative, respectively). For the 1 patient with the intraoperative neuromonitoring event, a wake-up test was performed and there were no postoperative deficits. None of the neurological complications required additional treatment.

\section{Discussion}

Anterior release and fusion of the thoracolumbar spine has been used for years in treating conditions such as severe scoliosis and kyphosis [16]. With advances in technology and improved posterior surgical techniques, the need for the anterior release in correcting spinal deformities has decreased.

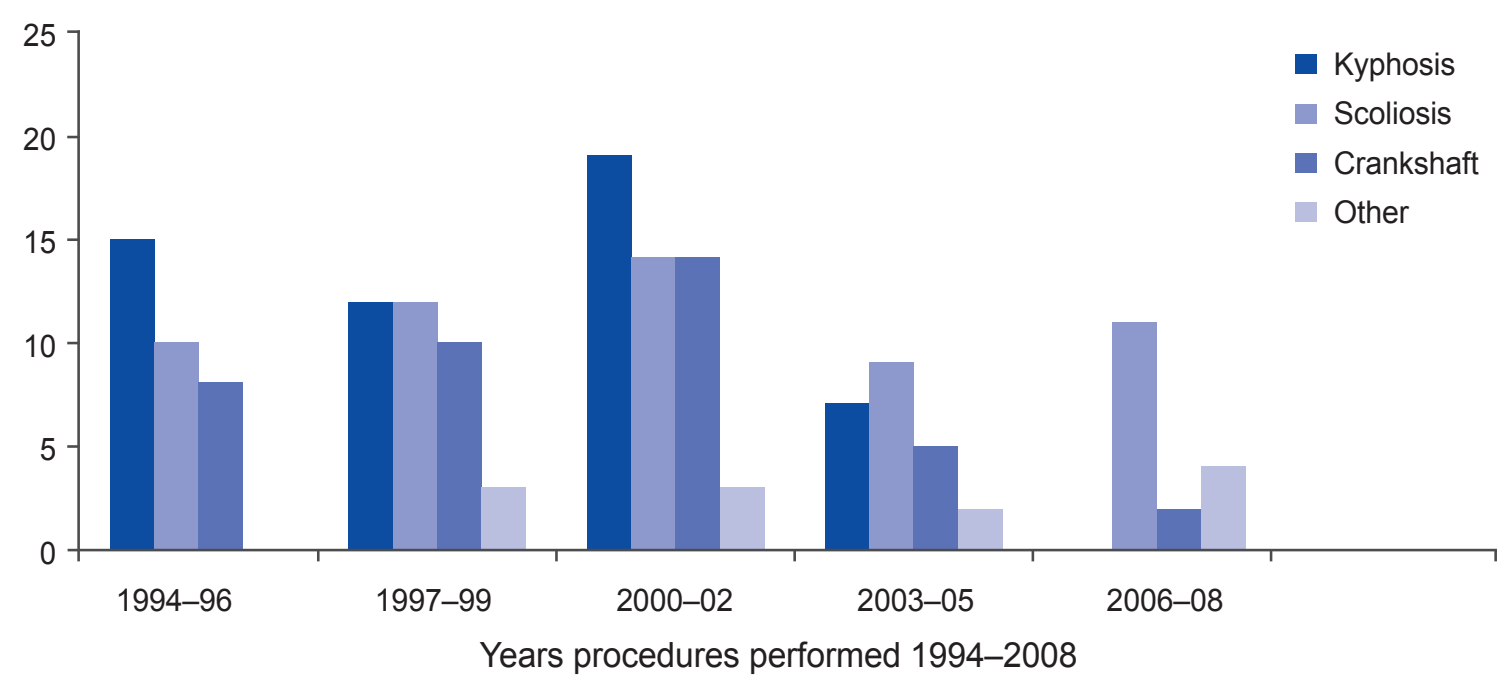

Fig. 2. Frequency of thoracoscopic anterior spinal fusion indications in 3-year intervals. 
We reviewed how the indications for anterior spinal fusion and instrumentation in pediatric spinal deformity patients have evolved at a single center between 1994 and 2008. Indications for the procedure were divided into 4 categories: Scheuermann kyphosis, crankshaft prevention, severe or rigid scoliosis, and other (e.g., pseudoarthrosis prevention, thoracic lordosis). While the number of spinal surgeries at our center has approximately doubled since 1994, the frequency of performing a thorcoscopic anterior release has decreased after reaching a peak in the years 2000-2002. During the initial period of the current study, Scheuermann kyphosis was the most frequent indication for an anterior spinal fusion and instrumentation. Since 2005, the increasing popularity of Ponte osteotomies and improved spinal instrumentation and fixation techniqueshas led to increased frequency of posterior only methods and a limited need for a combined anterior/posterior approach [17].

The use of thoracoscopy to prevent 'crankshaft' has also declined in recent years. The indication of crankshaft prevention with an anterior fusion peaked in 2000-2002, and is currently used for our most immature cases. The crankshaft phenomenon, as described by Dubousset et al. [18], is a progressive rotational deformity that results from the continued anterior spinal growth in the presence of a posterior fusion. Previous studies have shown that anterior arthrodesis is successful in preventing the crankshaft phenomenon [19-21].
Severe or rigid scoliosis is the most common current reason for performing a thoracoscopic release/fusion (Fig. 3). Several authors have recommended a combined anterior release/fusion and posterior fusion/instrumentation surgery to treat severe and rigid scoliotic deformities $[22,23]$; whereas others have recently suggested that a posterior-only procedure is sufficient $[14,15,24]$. Suk et al. [14] reported the use of a posterior only approach to treat severe scoliosis of $<110^{\circ}$. For more severe curves, an anterior approach or additional posterior techniques to supplement the posterior procedure remain an option [14].

We observed that since 1997, the anterior thoracoscopic procedure was used in other types of cases, such as pseudoarthrosis prevention and thoracic lordosis. Severe thoracic lordosis is a unique deformity that can be difficult to correct with a posterior-only procedure. Bradford et al. [25] argued that a combined approach is a valid procedure to restore normal thoracic kyphosis. Previous studies have suggested circumferential fusion for certain conditions or syndromes, such as Marfan syndrome or neurofibromatosis, because of their association with an increased risk of psedoarthrosis after spinal deformity correction surgery [26,27].

The anterior release procedure has largely been replaced by posterior-only techniques; however, we believe indications for the procedure remain due to the accepted advantages to using the thoracoscopic procedure, as compared
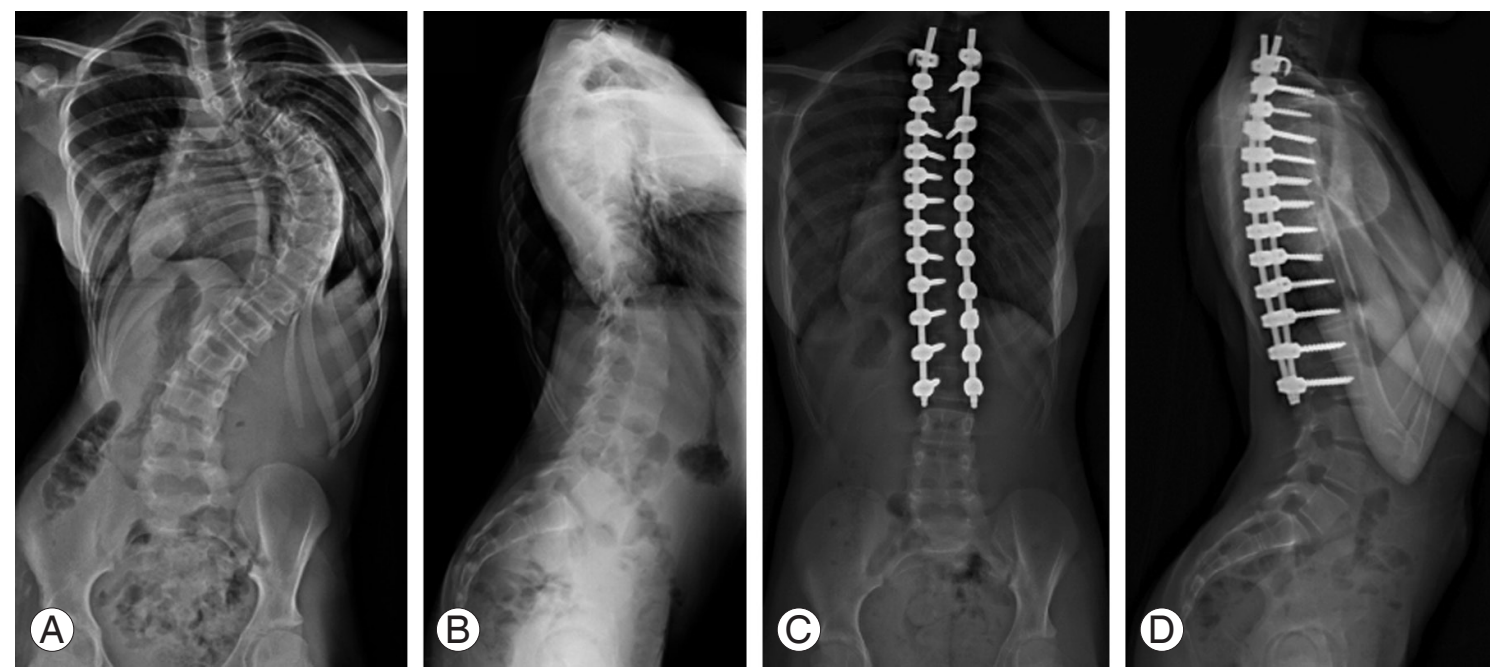

Fig. 3. Case example of 11 -year-old female, Risser 0, with Cobb angle of $98^{\circ}$ treated with an anterior thoracoscopic fusion from T5-T12 combined with a posterior spinal fusion from T2-L3 with segmental instrumentation. Ponte osteotomies were done from T6-L1. (A) Preoperative posteroanterior (PA) radiograph. (B) Preoperative lateral. (C) PA radiograph at 1 year postoperative. (D) Postoperative lateral. 
to an open thoracotomy. These advantages include excellent visualization of anatomical structures, less trauma to the tissues of the chest wall, less postoperative pain, and lower complication rates-particularly respiratory effects [28].

\section{Conclusions}

The indications for a thoracoscopic anterior release/fusion have evolved together with our understanding of this procedure and advances in posterior fixation with pedicle screw instrumentation. While less common than in the past decade, thoracoscopy still has an important role in select spinal deformity patients. However, the data does not allow precise current indication to be defined based on comparative data.

\section{Conflict of Interest}

No potential conflict of interest relevant to this article was reported.

\section{References}

1. Mack MJ, Regan JJ, Bobechko WP, Acuff TE. Application of thoracoscopy for diseases of the spine. Ann Thorac Surg 1993;56:736-8.

2. Rosenthal D, Rosenthal R, de Simone A. Removal of a protruded thoracic disc using microsurgical endoscopy: a new technique. Spine (Phila Pa 1976) 1994;19:1087-91.

3. Dickman CA, Rosenthal D, Karahalios DG, et al. Thoracic vertebrectomy and reconstruction using a microsurgical thoracoscopic approach. Neurosurgery 1996;38:279-93.

4. Horowitz MB, Moossy JJ, Julian T, Ferson PF, Huneke $\mathrm{K}$. Thoracic discectomy using video assisted thoracoscopy. Spine (Phila Pa 1976) 1994;19:1082-6.

5. Rosenthal D, Marquardt G, Lorenz R, Nichtweiss M. Anterior decompression and stabilization using a microsurgical endoscopic technique for metastatic tumors of the thoracic spine. J Neurosurg 1996;84:56572.

6. Newton PO. Thoracoscopic anterior instrumentation for idiopathic scoliosis. Spine J 2009;9:595-8.

7. Newton PO, Upasani VV, Lhamby J, Ugrinow VL, Pawelek JB, Bastrom TP. Surgical treatment of main thoracic scoliosis with thoracoscopic anterior instrumentation: surgical technique. J Bone Joint Surg Am 2009;91 Suppl 2:233-48.

8. Newton PO, Upasani VV, Lhamby J, Ugrinow VL, Pawelek JB, Bastrom TP. Surgical treatment of main thoracic scoliosis with thoracoscopic anterior instrumentation: a five-year follow-up study. J Bone Joint Surg Am 2008;90:2077-89.

9. Upasani VV, Newton PO. Anterior and thoracoscopic scoliosis surgery for idiopathic scoliosis. Orthop Clin North Am 2007;38:531-40.

10. Arlet V. Anterior thoracoscopic spine release in deformity surgery: a meta-analysis and review. Eur Spine J 2000;9 Suppl 1:S17-23.

11. Kostuik JP, Carl A, Ferron S. Anterior Zielke instrumentation for spinal deformity in adults. J Bone Joint Surg Am 1989;71:898-912.

12. Dwyer AF. Experience of anterior correction of scoliosis. Clin Orthop Relat Res 1973;(93):191-206.

13. Betz RR, Harms J, Clements DH 3rd, et al. Comparison of anterior and posterior instrumentation for correction of adolescent thoracic idiopathic scoliosis. Spine (Phila Pa 1976) 1999;24:225-39.

14. Suk SI, Kim JH, Cho KJ, Kim SS, Lee JJ, Han YT. Is anterior release necessary in severe scoliosis treated by posterior segmental pedicle screw fixation? Eur Spine J 2007;16:1359-65.

15. Burton DC, Sama AA, Asher MA, et al. The treatment of large (>70 degrees) thoracic idiopathic scoliosis curves with posterior instrumentation and arthrodesis: when is anterior release indicated? Spine (Phila Pa 1976) 2005;30:1979-84.

16. Bridwell $\mathrm{KH}$. Indications and techniques for anterior-only and combined anterior and posterior approaches for thoracic and lumbar spine deformities. Instr Course Lect 2005;54:559-65.

17. Geck MJ, Macagno A, Ponte A, Shufflebarger HL. The Ponte procedure: posterior only treatment of Scheuermann's kyphosis using segmental posterior shortening and pedicle screw instrumentation. J Spinal Disord Tech 2007;20:586-93.

18. Dubousset J, Herring JA, Shufflebarger H. The crankshaft phenomenon. J Pediatr Orthop 1989;9:541-50.

19. Shufflebarger HL, Clark CE. Prevention of the crankshaft phenomenon. Spine (Phila Pa 1976) 1991;16(8 Suppl):S409-11.

20. Dohin B, Dubousset JF. Prevention of the crankshaft 
phenomenon with anterior spinal epiphysiodesis in surgical treatment of severe scoliosis of the younger patient. Eur Spine J 1994;3:165-8.

21. Lapinksy AS, Richards BS. Preventing the crankshaft phenomenon by combining anterior fusion with posterior instrumentation. Does it work? Spine (Phila Pa 1976) 1995;20:1392-8.

22. Floman Y, Micheli LJ, Penny JN, Riseborough EJ, Hall JE. Combined anterior and posterior fusion in seventy-three spinally deformed patients: indications, results and complications. Clin Orthop Relat Res 1982;(164):110-22.

23. Johnson JR, Holt RT. Combined use of anterior and posterior surgery for adult scoliosis. Orthop Clin North Am 1988;19:361-70.

24. Dobbs MB, Lenke LG, Kim YJ, Luhmann SJ, Bridwell $\mathrm{KH}$. Anterior/posterior spinal instrumentation ver- sus posterior instrumentation alone for the treatment of adolescent idiopathic scoliotic curves more than 90 degrees. Spine (Phila Pa 1976) 2006;31:2386-91.

25. Bradford DS, Blatt JM, Rasp FL. Surgical management of severe thoracic lordosis: a new technique to restore normal kyphosis. Spine (Phila Pa 1976) 1983; 8:420-8.

26. Savini R, Cervellati S, Beroaldo E. Spinal deformities in Marfan's syndrome. Ital J Orthop Traumatol 1980; 6:19-40.

27. Tsirikos AI, Saifuddin A, Noordeen MH. Spinal deformity in neurofibromatosis type-1: diagnosis and treatment. Eur Spine J 2005;14:427-39.

28. Landreneau RJ, Hazelrigg SR, Mack MJ, et al. Postoperative pain-related morbidity: video-assisted thoracic surgery versus thoracotomy. Ann Thorac Surg 1993;56:1285-9. 